\title{
Experimental IRT applications in building elements with 3D thin defects
}

\author{
by N. Simões ${ }^{*, * *}$, C. Serra* and A. Tadeu*,** \\ *ITeCons - Institute for Research and Technological Development for Construction, Energy, Environment and \\ Sustainability, Rua Pedro Hispano s/ n., 3030-289 Coimbra, Portugal, nasimoes@itecons.uc.pt \\ ${ }^{*}$ ADAI - LAETA, Department of Civil Engineering, University of Coimbra, Pólo II, Rua Luís Reis Santos, \\ 3030-788 Coimbra, Portugal
}

\begin{abstract}
This paper presents an experimental study performed on building elements with embedded 3D thin defects looking to evaluate testing techniques used in Infrared Thermography (IRT) applications in buildings. An experimental campaign is performed on building elements with 3D defects located at different depths, using a pulse phase thermography based experimental setup. While numerous active IRT testing techniques have already been developed for Non-Destructive Testing (NDT) in many areas, there is still a substantial lack of active IRT applications in Civil Engineering due to the specific characteristics of building materials and construction solutions. This paper looks to further the application of experimental active IRT in buildings by presenting an experimental active IRT campaign which has been performed on test specimens simulating defected building elements containing thin inclusions beneath a ceramic tiling system. Test results are presented in terms of phase contrast curves.
\end{abstract}

\section{Introduction}

In the fields of aeronautics, electronics and mechanics, active Infrared Thermography (IRT) has been extensively and successfully employed as a Non-Destructive Testing (NDT) technique used to evaluate materials, products and equipments. Numerous active IRT testing techniques have been specifically developed for use in these areas [1]. However, due to the specific characteristics of building materials and construction solutions, nowadays there is still a substantial lack of active IRT applications in Civil Engineering. Among the most used active IRT techniques are pulse thermography, where a test specimen is flash heated and the resulting temperature decay is studied, and lock-in thermography where a sinusoidal stimulus is used and thermal wave phase results are obtained using a Fourier transform [2]. Analysing thermal wave phase results may present advantages over temperature results. Phase images are generally less affected by external factors such as reflections, surface emissivity changes and non-uniform surface heating. A phase contrast approach consists in calculating the difference between the thermal wave phase recorded in a defective area and in a sound area. The interpretation of phase contrast curves (representation of phase contrast in the frequency spectrum) enables the quantitative characterization of defects. Namely, defect depth retrieval techniques based on phase contrast curves by relating the defect's depth with the blind frequency (frequency at which the defect is no longer visible in terms of phase contrast images) or with the characteristic frequency (frequency at which the defect is most visible in terms of phase contrast images) have been proposed by authors [3],[4].

It has been suggested that pulse phase thermography, which is a combination of pulse thermography and lockin thermography, may be the most suited technique for Civil Engineering applications, since it allows enhanced defect detectability, better definition of the defect's geometry and inspections at greater depths [4]. For many years, the majority of thermographic studies in buildings have been performed with the buildings in their natural state, without making use of active IRT, and have been focused on evaluating energy efficiency and performing a qualitative assessment of the building envelope [5]. In constructions, depending on the local climate and the time of day, the radiation being emitted by surfaces and recorded using IRT varies greatly. Particularly in buildings, depending on the natural heating and cooling of the external surface via sun radiation and night-time cooling, as well as on the heating or cooling of the interior environment, there are many factors affecting IRT measurements. If there is a sufficient temperature difference between interior and exterior then inspections in buildings may be performed in a passive manner with its surfaces in their natural state, however, the interpretation of results may vary on account of the environmental conditions during the inspection. Whether the inspection is being performed on the exterior envelope of a building or inside, if an additional stimulus is necessary to produce the temperature differences required for defect detection, then an active IRT technique must be employed. Since there is a growing need for monitoring and assessing the energy performance of buildings, as well as for evaluating the thermal behavior of buildings' envelopes and the structural safety of constructions, the intensification of the use of quantitative IRT studies in buildings inspections is strongly supported. As a result, studies have been performed using active IRT to evaluate concrete and masonry structures [6], [7] and to characterize the hygrothermal behavior of building elements [8], showing promising results.

There is great potential in using IRT in ceramic tiling systems to evaluate their integrity. This kind of building solution often present durability problems (detachment, cracking, efflorescence) which are generally caused by poor adhesion at the time of application of the ceramic tiles. If they are not applied adequately, hollow areas between the adhesive layer and the ceramic tile or between the adhesive layer and the substrate may become filled with water (from rain or condensation) leading to the premature deterioration of materials and eventually to the adhesive failure and 
detachment of the ceramic tiles [9],[10]. Since tiles and concrete spalling falling out from external walls in buildings represent serious risks to people's safety, it is necessary to detect and assess tiling application anomalies before adhesive failure occurs. Since hollow areas appear in thermographic images, IRT is a non-destructive technique which may be used to detect and assess these types of problems.

Looking to further the application of experimental active IRT in buildings and evaluate IRT testing techniques, the present paper presents an experimental active IRT study performed on test specimens of defected building elements with thin inclusions placed behind a ceramic tile, using a phase contrast approach.

\section{Methodology}

In this paper, an experimental campaign is performed on test specimens simulating building elements containing thin 3D defects with varying properties and thicknesses, and located at two different depths beneath a ceramic material. The active thermography tests are performed using a pulse phase thermography based experimental setup (long pulse) and some experimental parameters are varied in order to assess their influence in the results. The test results are presented in terms of phase contrast curves.

Phase contrast is the difference between the thermal wave phase recorded in a defective area and in a sound area. Null phase contrast indicates a sound area, while the existence of phase contrast indicates the presence of a defect. A phase contrast curve can be extracted for any pixel within the field of view (image captured by an infrared camera in IRT tests) and contains useful information about the soundness of the particular zone being inspected for defects. In a phase contrast curve maximum absolute phase contrast is reached at the characteristic frequency $f_{c h}$ and the frequency at which phase contrast becomes null is known as the blind frequency $f_{b}$.

In these experiments phase results are calculated for specific pixels by applying a Fourier transform to the thermal wave results recorded in the time domain. For each pixel, phase contrast is obtained by computing the difference between the recorded thermal wave phase and the phase recorded at an area which is not influenced by the defect (sound zone).

\section{Experimental Campaign}

\subsection{Experimental Apparatus}

Active IRT tests are performed using a FLIR A615 camera connected to a control unit (Automation Technology IRX-Box) and a computer with IR NDT 1.74 software. The IRT camera has the following technical specifications: pixels resolution of $640 \times 480$; spectral range of $7.514 \mu \mathrm{m}$; field of view (FOV) of $25^{\circ} \times 19^{\circ}$; spatial resolution of $0.68 \mathrm{mrad}$; thermal sensitivity/noise equivalent temperature difference (NETD) $<0.05{ }^{\circ} \mathrm{C} @ 30{ }^{\circ} \mathrm{C}$ and accuracy of $\pm 2{ }^{\circ} \mathrm{C}$ or $2 \%$. The tests were performed in reflection mode with both the camera and the heat source placed $1.27 \mathrm{~m}$ away from the surface of the test specimen and using two $2500 \mathrm{~W}$ halogen. The thermal stimulation follows a rectangular function. In each test, thermal images were recorded for a defined length of time (test duration), which includes an initial offset period, a heating period and a cooling down period.

\subsection{Test Specimen}

Two test specimens were prepared in order to simulate defected building elements with two ceramic tiling products with different thickness. A schematic representation of the test specimens, along with the location of the defects, is given in Figure 1.

Each test specimen follows a multilayered configuration and contains six inclusions (or defects). The inspected surface is a layer of ceramic material with varying thickness $(5 \mathrm{~mm}$ and $11 \mathrm{~mm})$. This is followed by a layer of mortar, and a layer of insulation material is placed at the back of the specimen in order to minimize heat flow. The thin inclusions are made from different materials (agglomerate cork board and polyethylene foam with varying thickness and density) and are placed between the ceramic material and the mortar. In the upper row of defects, the inclusions are made from polyethylene foam ( $A$ is $2.2 \mathrm{~mm}$ thick, $B$ is $6.01 \mathrm{~mm}$ and $C$ is $10.30 \mathrm{~mm}$ ). Bellow the defects are agglomerate cork board ( $D$ is $2.18 \mathrm{~mm}, E$ is $4.13 \mathrm{~mm}$ and $F$ is $10.29 \mathrm{~mm}$ ). 


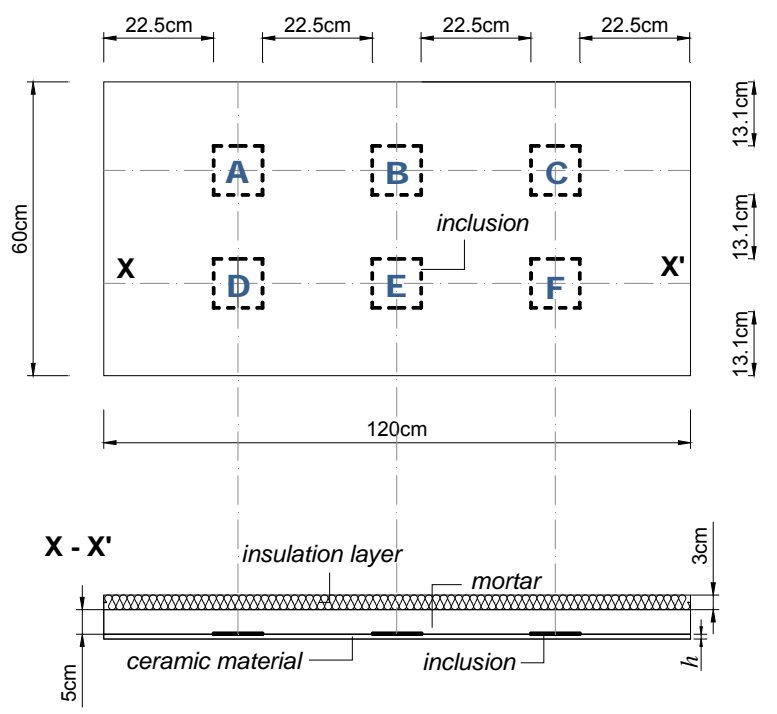

Fig. 1. Front view and cross section of the test specimen.

\subsection{Results}

\subsubsection{Influence of defect depth}

Using the Fourier Transform to process the experimental data, test results are extracted in terms of phase contrast curves. Figure 2 shows the experimental phase contrast results for the test specimen with the thinner ceramic material subject to a test where 128 thermal images were recorded for a $4096 \mathrm{~s}$, in which, after an initial offset period of $100 \mathrm{~s}$, the surface was heated for 200 s using $100 \%$ of heating power $(2 \times 2500 \mathrm{~W})$. In the lower frequencies, these graphic show a distinct curve for each defect, however, in the higher frequencies they no longer follows a distinct curve and even oscillate between negative and positive phase-contrast values. This can be attributed to the presence of noise in the phase results.

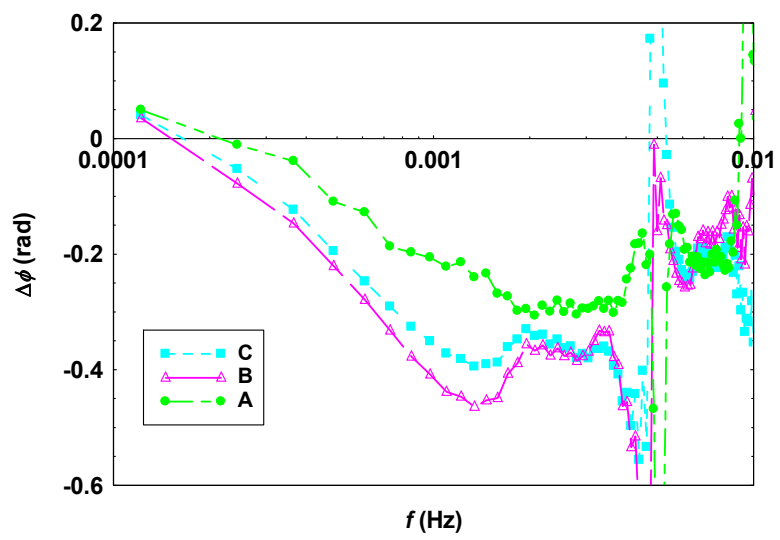

a)

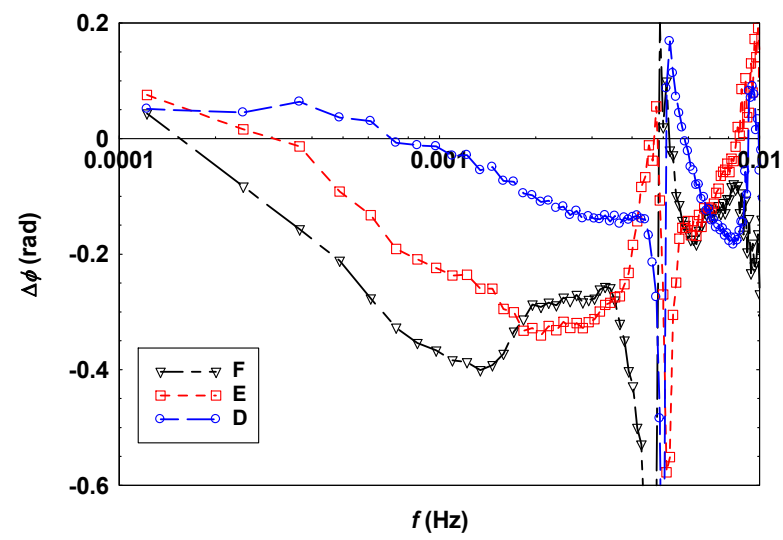

b)

Fig. 2. Experimental phase contrast results for inclusions located beneath a ceramic material with a thickness of $5 \mathrm{~mm}$ : a) inclusions $A, B$ and $C$; $b$ ) inclusions $D, E$ and $F$.

In the graph in Figure 2.b) it is clear that the thickest agglomerate cork inclusion (F) produces the greatest disturbance in terms of propagation of thermal waves within the specimen and its curve shows a clearly defined peak. Also, the thinnest agglomerate cork inclusion (D) produces the least disturbance, in fact, even though there is visible phase contrast, there is no defined peak in phase contrast. On the other hand, in Figure 2.a), the polyethylene foam defect $B$ is the one with greater phase contrast. This may be due the varying diffusivity of the materials used as defects. Figure 3 illustrates the phase contrast results obtained for a specimen with a thicker ceramic tile subject to a $4096 \mathrm{~s}$ test (offset period of 100 s, heating time of 200 s, $100 \%$ heating power) during which 128 frames where recorded. 


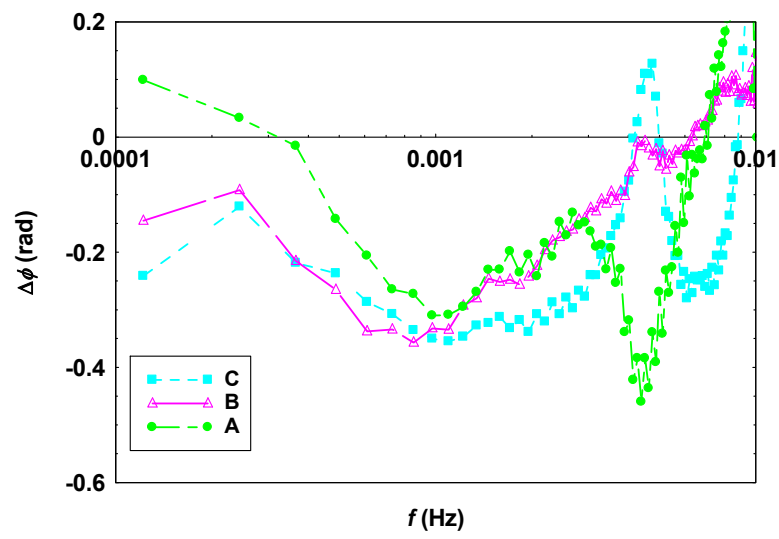

a)

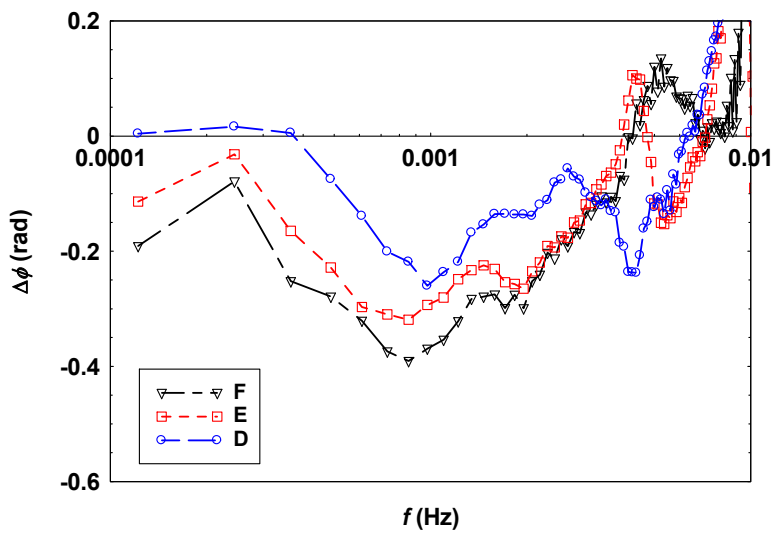

b)

Fig. 3. Experimental phase contrast results for inclusions located behind a ceramic material with a thickness of $11 \mathrm{~mm}$ : a) inclusions $A, B$ and $C$; $b$ ) inclusions $D, E$ and $F$.

By comparing Figure 2 and Figure 3 it can be observed that when increasing the depth at which a defect is located the resulting curves are slightly shifted to the left, hence resulting in a decrease in both the characteristic frequency and the blind frequency, which is in accordance with the claim that defect depth is inversely related to $f_{b}$ and $f_{c h}[3],[4]$.

\subsubsection{Influence of test setup parameters}

The testing parameters defined for IRT experiments in building elements have great influence on the phase contrast results and therefore on the interpretation of phase contrast results. The following graphs illustrate the importance in correctly defining parameters, namely heating duration and heating power, in order to obtain sufficient recording time and frequency resolution.

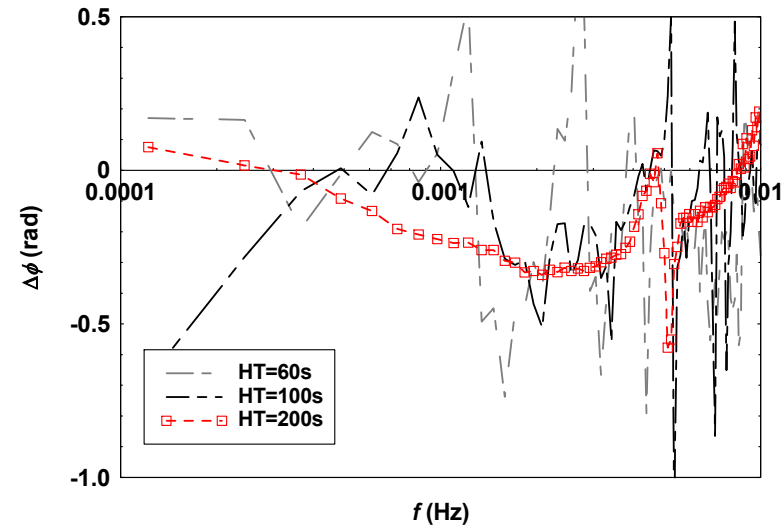

Fig. 4. Influence of heating time (HT) on experimental phase contrast results for inclusion $E$ located behind a ceramic material with a thickness of $5 \mathrm{~mm}$.

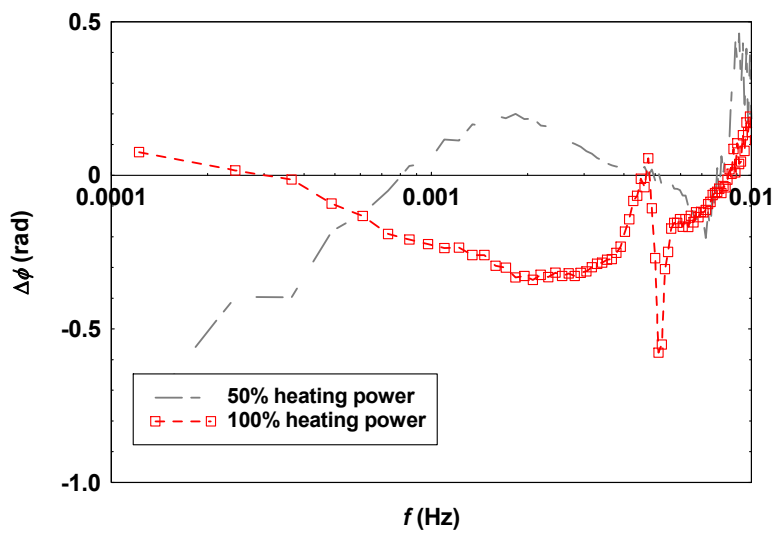

Fig. 5. Influence of heating power on experimental phase contrast results for inclusion E located behind a ceramic material with a thickness of $5 \mathrm{~mm}$.

\section{Conclusions}

In this paper an experimental campaign was performed to evaluate defects placed beneath a ceramic tiling system using a pulse phase based active IRT technique. The experimental data was processed using a Fourier transform in order to generate phase contrast results. The tests conducted allowed the detection of all of the defects present in both of the test specimens. Phase contrast curves were extracted for specific pixels of the defected areas within the field of view of the IRT camera. These revealed that, in each specimen, the various defects located at the same depth generated different levels of phase contrast amplitude, which can be attributed to the difference in the nature 


\subsection{1/qirt.2016.161}

of the materials as well as their thickness. Thicker defects generally produced a greater disturbance in the heat field, therefore generating greater phase-contrast.

The experimental setup was based in previous work conducted by the authors [11]. Test parameters such as heating time and heating power are decisive for the generation of phase contrast curves. The curves obtained from these experiments showed a peak in phase-contrast, however the definition of the characteristic frequency $f_{c h}$ and the blind frequency $f_{b}$ were not sufficiently clear so as to successfully employ a depth inversion technique.

Future work will focus on varying the frequency resolution and acquisition time and on improving the data sampling techniques in order to define the optimal experimental setup for quantitative evaluations of defected building elements using active IRT.

\section{AKNOWLEDGEMENTS}

This work has also been supported by the POCl-01-0247-FEDER-003179 (Revi Clean Facade) Project funded by Portugal 2020 through the Operational Programme for Competitiveness Factors (COMPETE 2020).

\section{REFERENCES}

[1] Maldague X., "Theory and Practice of Infrared Technology for Non-destructive Testing". John Wiley \& Sons, 2001.

[2] Maldague X., Introduction to NDT by Active Infrared Thermography, Materials Evaluation, Vol. 6, no. 9 (2002), pp.1060-1073.

[3] Clemente Ibarra-Castanedo \& Xavier Maldague (2004) Pulsed phase thermography reviewed, Quantitative InfraRed Thermography Journal, 1:1, 47-70, DOI: 10.3166/qirt.1.47-70

[4] Arndt R.W., Square pulse thermography in frequency domain as adaptation of pulsed phase thermography for qualitative and quantitative applications in cultural heritage and civil engineering, Infrared Physics \& Technology, Vol. 53, no.4 (2010), pp.246-253.

[5] Balaras C.A., Argiriou A.A., Infrared thermography for building diagnostics, Energy and Buildings, Vol. 34 (2002), pp.171-183

[6] Meola C., Infrared thermography of masonry structures, Infrared Physics \& Technology, Vol. 49 (2006), pp.228-233.

[7] Maierhofer Ch., Brink A., Röllig M., Wiggenhauser H., Transient thermography for structural investigation of concrete and composites in the near surface region, Infrared Physics \& Technology, Vol. 43, no.3 (2002), pp.271-278.

[8] Laurenti L., Marcotullio F., Monte F., Determination of the thermal resistance of walls through a dynamic analysis of in-situ data, International Journal of Thermal Science, Vol. 43 (2004), pp.297-306.

[9] Edis E., Flores-Colen I., de Brito J., "Passive thermographic inspection of adhered ceramic claddings: limitation and conditioning factors". Journal of Performance of Constructed Facilities, vol. 27(6), pp.737 747, 2013.

[10] Li Z., Yao W., Lee S., Lee C., Yang Z., "Application of infrared thermography technique in building finish evaluation". Journal of Nondestructive Evaluation, vol. 19(1), pp. 11-19, 2000.

[11] Serra C., Simões N., Tadeu A., (2015) Study of experimental parameters for active IRT applications in multilayered building elements with internal defects, Thermosense: Thermal Infrared Applications XXXVI, 20-24 April, Baltimore, MD, USA. 